\title{
Hybrid Reasoning Over Large Knowledge Bases Using On-The-Fly Knowledge Extraction
}

\author{
Giorgos Stoilos (D), Damir Juric, Szymon Wartak, Claudia Schulz ${ }^{(凶)} \mathbb{( D}$, \\ and Mohammad Khodadadi \\ Babylon Health, London, SW3 3DD, UK \\ \{giorgos.stoilos, damir.juric, szymon.wartak, claudia.schulz, \\ mohammad.khodadadi\}@babylonhealth.com
}

\begin{abstract}
The success of logic-based methods for comparing entities heavily depends on the axioms that have been described for them in the Knowledge Base (KB). Due to the incompleteness of even large and well engineered KBs, such methods suffer from low recall when applied in real-world use cases. To address this, we designed a reasoning framework that combines logic-based subsumption with statistical methods for on-the-fly knowledge extraction. Statistical methods extract additional (missing) axioms for the compared entities with the goal of tackling the incompleteness of KBs and thus improving recall. Although this can be beneficial, it can also introduce noise (false positives or false negatives). Hence, our framework uses heuristics to assess whether knowledge extraction is likely to be advantageous and only activates the statistical components if this is the case. We instantiate our framework by combining lightweight logic-based reasoning implemented on top of existing triplestores with an axiom extraction method that is based on the labels of concepts. Our work was motivated by industrial use cases over which we evaluate our instantiated framework, showing that it outperforms approaches that are only based on textual information. Besides the best combination of precision and recall, our implementation is also scalable and is currently used in an industrial production environment.
\end{abstract}

Keywords: Large medical ontologies • Axiom extraction from text • Hybrid reasoning

\section{Introduction}

Large Knowledge Bases (KBs) have started to play a key role in applications like dialogue systems [29], healthcare [4], and recommendation systems [20]. KBs describe the entities of the domain at hand and their relationships. This enables semantic interpretation of information expressed in terms of knowledge from a

G. Stoilos-Since submission Giorgos Stoilos is a member of Huawei Technologies, R\&D, UK. 
$\mathrm{KB}$, and thus allows the exchange of information between different systems or components expressing information using a shared KB.

To capture real-world meaning, systems may not only use atomic concepts from the KB but also combine concepts to express more complex entities of the domain. For example, in a biomedical application using SNOMED $\mathrm{CT}$ to represent medical data, a user profile may contain the concept $C_{1}:=$ RecentInjury $\sqcap \exists$ findingSite.Head, capturing the condition "recent injury in the head" associated with the patient. Yet, in a symptom-checking service the same condition may be represented as $C_{2}:=$ Headlnjury $\sqcap \exists$ occurred.Recent. To enable proper information exchange between these services, subsumption reasoning over the stored knowledge is crucial to correctly match equivalent information expressed in different ways, i.e. to prove that $\mathcal{K} \models C_{1} \sqsubseteq C_{2}$ and $\mathcal{K} \models C_{2} \sqsubseteq C_{1}$ for a $\mathrm{KB} \mathcal{K}$.

In theory, ontology reasoners like ELK [15] can be used for this kind of subsumption checking, however, in practice this is problematic for at least two reasons. First, even large and well-engineered KBs suffer from incompleteness [10,21], leading to very low recall of equivalent and subsumed concepts. Indeed, for the above example and for $\mathcal{K}$ the SNOMED CT, we have $\mathcal{K} \forall C_{1} \sqsubseteq C_{2}$ and $\mathcal{K} \forall C_{2} \sqsubseteq C_{1}$ since $\mathcal{K}$ is missing an axiom of the form $a x:=$ RecentInjury $\equiv$ Injury $\sqcap \exists$ occurred.Recent. SNOMED CT contains many such ill-defined concepts [21] like SevereDepression, which is not defined in terms of concepts Severe and Depression, or CardiacMuscleThickness, not defined in terms of CardiacMuscle and Thick. The same can be observed for other KBs like DBpedia [1], where the category ItalianRenaissancePainters is not connected to concepts Painter or ItalianRenaissance. Second, such reasoners are not designed for reasoning over very large industrial KBs, like DBpedia and Freebase, which are usually stored in triple-stores or graph databases.

To address the above issues, we designed a hybrid reasoning framework for subsumption checking that couples logic-based reasoning with on-the-fly knowledge extraction. Knowledge extraction is used to enrich the concepts being compared with intended but missing axioms and hence increase recall of subsumption checking. Since knowledge extraction can produce false positives, i.e. lead to subsumptions that are not intended, the framework incorporates heuristics to decide when to apply knowledge extraction.

We give a concrete instantiation of our framework, where the knowledge extraction component is realised using Natural Language Processing techniques that construct (complex) concepts from the labels of the compared concepts. For instance, in our running example, the label of concept Recentlnjury in SNOMED $\mathrm{CT}$ is "Recent Injury". From that, our knowledge extraction component constructs the more detailed concept Injury $\sqcap$ ヨoccurred.Recent, thus recovering the missing axiom $a x$ and eventually enabling to prove the subsumption since $\mathcal{K} \cup\{a x\} \models C_{1} \sqsubseteq C_{2}$. Furthermore, to allow reasoning over large industrial KBs we realise logic-based reasoning via a lightweight approximate reasoner implemented on top of existing triple-stores. Finally, we present concrete instantiations of the heuristics controlling the knowledge extraction component. 
Importantly, our knowledge extraction method not only forms a crucial part of the hybrid reasoning framework, but it can also be applied as a stand-alone method for constructing complex concepts from short phrases and can thus be useful, e.g., for keyword-based and entity-centric query answering on top of KBs $[13,14,23]$. This furthermore enables us to apply our hybrid reasoner to textual inputs (short phrases), which can be transformed into concepts using the stand-alone knowledge extraction component, followed by the normal application of our hybrid reasoner.

Our work was motivated by several industrial use cases in Babylon Health ${ }^{1}$, a digital health care provider offering services such as AI-based symptom-checking and triaging. Data in all services are encoded using (complex) concepts built from a large medical KB that is stored in a triple-store. It is desirable that different services exchange and compare concepts for the purposes of interoperability, ensuring the delivery of intelligent services to end-users. For example, if new evidence for a "head injury" is encoded in the user profile in the form of concept $C_{1}$, other services like triaging or symptom-checking need to be able to interpret $C_{1}$ and compare it to concepts used by these services, e.g. $C_{2}$, to provide advice to the patient on how to proceed. We tested our implementation in two use cases within this industrial healthcare setting. Our results show the advantage of our knowledge extraction method used internally in our hybrid subsumption checking reasoner compared to pure logic-based reasoning, as well as the high quality of concepts constructed by the extraction method. Furthermore, results reveal that our hybrid reasoner provides the best combination of precision and recall compared to approaches that are purely statistical or purely rule-based.

\section{Preliminaries}

The concept extraction method we design will construct concepts expressed in the Description Logic (DL) $\mathcal{E} \mathcal{L}[2]$, that is, concepts that are defined by the grammar $C:=\top|A| C_{1} \sqcap C_{2} \mid \exists R . C$ where $A$ is an atomic concept and $R$ is an atomic property. In addition to the standard DL notation, we assume that every atomic concept $A$ (resp. property $R$ ) has an associated label denoted by $\mathscr{L}(A)$ (resp. $\mathscr{L}(R)) . \mathcal{E} \mathcal{L}$-concepts can also be written as $\prod_{i} A_{i} \sqcap \prod_{j} \exists R_{j} . C_{j}$ where each $A_{i}$ is an atomic concept or $\top$ and each $C_{j}$ is again an $\mathcal{E} \mathcal{L}$-concept. We also consider concepts like $\exists R . B$ to be of the form $\top \sqcap \exists R . B$.

$\mathcal{E} \mathcal{L}$ is expressive enough to capture many medical KBs like SNOMED CT. In fact, as noted in [8], the SNOMED CT KB is primitive, that is, it can be rewritten as a set of subsumption axioms of the form $A \sqsubseteq D$ where $A$ is atomic. This is because full definitions of the form $A \equiv D$ are acyclic and therefore can be recursively unfolded into axioms of the form $B \sqsubseteq \exists R$. $A$ to obtain $B \sqsubseteq \exists R . D$ and then discard $A \equiv D$.

The reasoning problem investigated in our work is subsumption checking between two concepts $C$ and $D$ of the above form with respect to a KB $\mathcal{K}$ - that

\footnotetext{
${ }^{1}$ https://www.babylonhealth.com/.
} 
is, whether $\mathcal{K}=C \sqsubseteq D$. However, acknowledging the fact that KBs are incomplete $[10,21]$ the problem we study is whether a set of "missing" axioms $\mathcal{K}^{\prime}$ can be extracted such that $\mathcal{K} \cup \mathcal{K}^{\prime} \mid=C \sqsubseteq D$. In order not to modify the KB during subsumption checking, the axioms in $\mathcal{K}^{\prime}$ can be immediately unfolded into $C$ and $D$, obtaining new concepts $C^{\prime}$ and $D^{\prime}$. We thus reformulate the original axiom addition problem as a concept rewriting problem and check if $\mathcal{K} \models C^{\prime} \sqsubseteq D^{\prime}$.

\section{A Framework for Hybrid Reasoning}

In theory, given two concepts standard reasoning techniques can be used to compare them w.r.t. subsumption. However, the success of this task heavily depends on the knowledge described in the KB for every concept and, as already pointed out in the literature [21], a lot of relevant knowledge is usually missing.

Example 1. Consider the SNOMED CT KB $\mathcal{K}$ and the phrase "recent head injury", which different services may represent in the following different ways:

$C_{1}:=$ RecentInjury $\sqcap \exists$ findingSite. Head

$C_{2}:=$ Injury $\sqcap \exists$ findingSite. Head $\sqcap \exists$ occurred.Recently

It can be verified using any DL reasoner that $\mathcal{K} \forall C_{1} \sqsubseteq C_{2}$ and $\mathcal{K} \forall$ $C_{2} \sqsubseteq C_{1}$ since $\mathcal{K}$ is missing an axiom of the form Recentlnjury $\equiv$ Injury $\sqcap$ $\exists$ occurred.Recently.

The above example highlights the need for a method that tries to construct such missing but intended axioms for any two concepts to enable higher recall of traditional subsumption checking algorithms. Looking again at the example, we note that the missing knowledge is in fact encoded in the label of the concept Recentlnjury, which is "recent injury". More precisely, using this label, our goal is to construct an axiom $a x:=$ RecentInjury $\equiv$ Injury $\sqcap$ Joccurred.Recently and then directly unfold it into $C_{1}$ for subsumption checking, yielding $C_{1}^{\prime}:=\operatorname{Injury} \sqcap$ $\exists$ occurred.Recently $\sqcap \exists$ findingSite. Head. We would then have $\mathcal{K} \models C_{1}^{\prime} \sqsubseteq C_{2}$ and $\mathcal{K} \models C_{2} \sqsubseteq C_{1}^{\prime}$, implying the equivalence of $C_{1}$ and $C_{2}$ (given the missing axiom).

Although axiom extraction and concept unfolding can improve recall of subsumption checking, they may also introduce false positives (i.e. subsumption of concepts that should be unrelated) or even false negatives (i.e. failure of proving subsumption for concepts that are subsumed without unfolding).

Example 2. Assume that for the concepts in Example 1, we also use the label of concept Injury to unfold $C_{2}$, which is "Traumatic AND/OR non-traumatic injury". A concept extraction method is likely to construct an axiom $a x^{\prime}:=$ Injury $\equiv$ Injury $\sqcap \exists$ assocWith. Traumatic $\sqcap \exists$ assocWith.NonTraumatic from this. Then, for $C_{2}^{\prime}$ the unfolding of $a x^{\prime}$ in $C_{2}$, we will have $\mathcal{K} \not C_{1}^{\prime} \sqsubseteq C_{2}^{\prime}$.

Based on the above, we designed a hybrid reasoning framework, given in Algorithm 1, which combines logic-based subsumption checking with on-the-fly statistical knowledge extraction. Given a candidate subsumption $C \sqsubseteq D$, the algorithm first attempts to use standard logic-based reasoning (line 1) to prove 


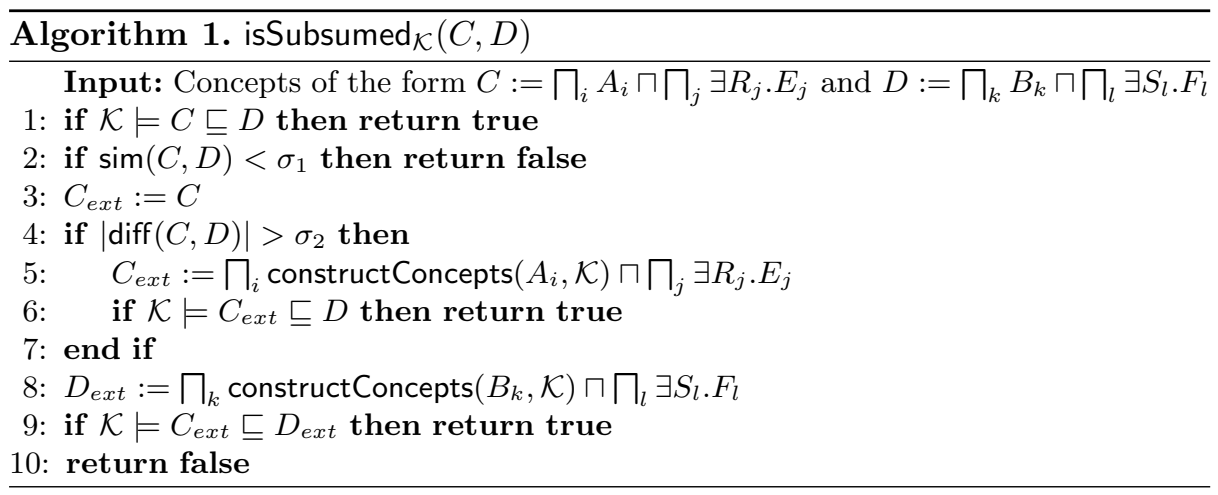

subsumption over a KB $\mathcal{K}$. If subsumption cannot be proven, the knowledge extraction method may be activated, subject to some applicability conditions. The first condition (sim) assesses if the similarity of the compared concepts $C$ and $D$ is sufficiently high (line 2). Intuitively, the higher the similarity, the more likely it is for the axiom extraction step to lead to true positive results.

If the first condition is satisfied, the question is which of the two concepts should be extended with additional knowledge. Due to the monotonicity of the OWL semantics, adding axioms for $C$ would be valuable since failure to prove subsumption implies that $D$ likely has "more constraints" (conjuncts) than $C$. These additional axioms could then make up for the missing constraints. The second applicability condition (diff) thus checks whether $D$ has more constraints than $C$. If so, the knowledge extraction component is activated to unfold $C$ with an axiom obtained by processing its main atomic concepts (line 5 ). If the unfolded $C$ is still not subsumed by $D, D$ is also unfolded (line 8 ).

There are various things to note about our hybrid reasoning framework. First, extraction is only applied on the main atomic concepts (i.e. the $A_{i}$ and $B_{k}$ ) as these represent the gist of a complex concept. Applying knowledge extraction on more parts of a complex concept would induce further fuzziness, thus increasing the probability for false positives and false negatives. For the same reason, our hybrid reasoner applies knowledge extraction on-the-fly instead of extracting all possible axioms from concept labels before reasoning, which would lead to the unrestricted usage of extracted axioms. For small KBs, extracting axioms upfront could allow manual validation, thus providing an advantage over on-thefly extraction. However for large-scale industrial KBs as investigated here, this is infeasible since there is no way to reliably validate thousands of axioms. Thus, on-the-fly extraction is more beneficial for such KBs.

The exact implementation used for logic-based subsumption checking and knowledge extraction is up to the user and depends on the application at hand. In the following sections, we present concrete instantiations of all parts, targeted at knowledge extraction from concept labels and reasoning over triple-stores. 


\section{Extracting Concepts from Text}

Our concept extraction method (i.e., the implementation of constructConcepts in Algorithm 1) will be based on the labels of concepts. Labels have been used in the past for tasks like ontology enrichment $[9,21]$ as they constitute a good source of additional knowledge that is usually in abundance in ontologies.

The problem of constructing concepts from their labels can be broken down into two parts, as further detailed in the following sections: 1) parsing the label to correctly identify its parts, and 2) linking each part to atomic concepts in the $\mathrm{KB}$ and selecting appropriate KB properties to piece them together.

\subsection{Parsing the Phrase Structure}

Concept labels usually follow a specific pattern, that is, they contain a central entity, frequently narrowed down by modifiers. For example, in the SNOMED CT concept label "pain in left leg" the central entity is "pain", which is further detailed by "leg", which in turn is narrowed down via modifier "left". Examples of concept labels in other KBs are "Italian Renaissance painters" or "Thriller Movie". Such phrases may include linguistic constructs like prepositions, e.g., "Pain in Leg" versus "Leg Pain", and can in some rare cases contain verbs, e.g., "central sleep apnea caused by high altitude". Finally, KBs seldom contain concepts representing conjunctions or disjunctions of atomic entities, hence their labels rarely include coordinating conjunctions like "pain in arm and chest" and never contain non- $\mathcal{E} \mathcal{L}$ constructors like "at least" and "for all".

The above observations motivate the use of dependency parsing [16], which precisely attempts to capture the main word in a phrase (called root) and then recursively append the rest of the modifiers to it. At an abstract level, the dependency tree of a phrase txt can be characterised as a tree where each node nd is labelled with a corresponding word from txt, denoted nd. $\ell$, and each edge $\left\langle\mathrm{nd}_{1}, \mathrm{nd}_{2}\right\rangle$ is labelled with a dependency relation $\left\langle\mathrm{nd}_{1}, \mathrm{nd}_{2}\right\rangle . \ell$ that denotes the linguistic relation between the nodes $\mathrm{nd}_{1} . \ell$ and $\mathrm{nd}_{2} . \ell$.

Figure 1a depicts the dependency tree of the phrase "recent pain caused by injury" obtained using ClearNLP [6]. Since a dependency parser constructs a

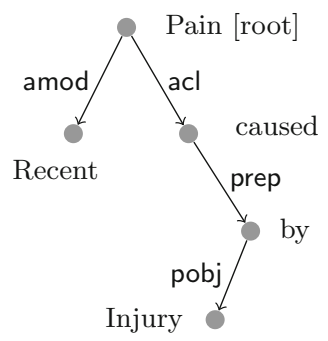

(a) Dependency Tree

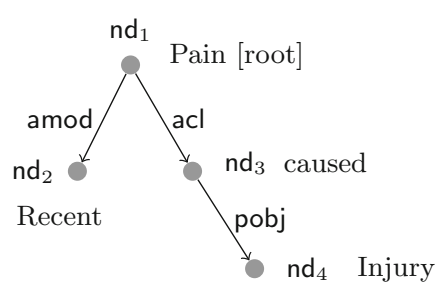

(b) Normalised Tree

Fig. 1. Dependency and normalised trees of "recent pain caused by injury". 
node for every word in the phrase, compound concepts like "heart attack" are split into separate nodes. However, grouping them is beneficial for better interpreting meaning [27]. Moreover, since prepositions do not carry concept meaning, we prune prepositions and other functional words. In summary, the dependency tree is post-processed as follows:

$(\diamond)$ All paths $\left\langle\mathrm{nd}_{1}, \mathrm{nd}_{2}\right\rangle, \ldots,\left\langle\mathrm{nd}_{n-1}, \mathrm{nd}_{n}\right\rangle$ with $n \geq 2$ such that each edge is labelled with dependency relation compound are collapsed to one node $\mathrm{nd}_{1}$ with new label $\mathrm{nd}_{1} . \ell \oplus "$ " $\oplus \ldots \oplus$ " " $\oplus \mathrm{nd}_{n} . \ell$ where $\oplus$ denotes string concatenation.

$(\sharp)$ All paths $\left\langle\mathrm{nd}_{1}, \mathrm{nd}_{2}\right\rangle, \ldots,\left\langle\mathrm{nd}_{n-1}, \mathrm{nd}_{n}\right\rangle$ with $n \geq 2$ such that each edge is labelled with either prep or case are collapsed to one node $\mathrm{nd}_{1}$.

Due to $(\sharp)$ the dependency tree in Fig. 1a is reduced to the tree in Fig. 1b. This post-processing can be easily realised using a tree traversal algorithm.

\subsection{Building Concepts}

After parsing the phrase to identify the important parts, atomic concepts from the $\mathrm{KB}$ need to be linked to the tree nodes, a problem referred to as entity linking [12]. Furthermore, a KB property between them needs to be established in order to construct a (complex) concept.

Many approaches have been proposed for entity linking, most of them involving supervised machine learning methods [24]. We use a simple, yet effective and scalable, information retrieval approach, which does not require a large training dataset. More precisely, given the label nd. $\ell$ of a node in the tree, ElasticSearch is used to search the KB for the concept or property with the most similar label, denoted by $\operatorname{linkC}(\ell)$ and $\operatorname{linkR}(\ell)$, respectively. If no matching concept can be found, linkC $(\ell)=\perp$, and if no matching property can be found, linkR $(\ell)=$ assocWith, using the most common and versatile property.

A concept corresponding to a normalised dependency tree is defined recursively as given in Definition 1. We distinguish two strategies for obtaining a property between two concepts. If the tree contains three adjacent nodes $\mathrm{nd}_{i}$, $\mathrm{nd}_{j}$, and $\mathrm{nd}_{k}$ such that $\mathrm{nd}_{j}$ is a verb, linkR links the verb to a property in the KB to connect the concepts represented by nodes $\mathrm{nd}_{i}$ and $\mathrm{nd}_{k}$; otherwise, a property is "mined" using two adjacent nodes representing concepts.

Definition 1. For a node $\mathrm{nd}_{i}$ in some dependency tree, the concept corresponding to $\mathrm{nd}_{i}$ is $\perp$ if linkC $\left(\mathrm{nd}_{i} . \ell\right)=\perp$, otherwise it is recursively defined as follows:

$$
\begin{aligned}
\operatorname{linkC}\left(\mathrm{nd}_{i} \cdot \ell\right)= & \prod_{\left\langle\mathrm{nd}_{i}, \mathrm{nd}_{j}\right\rangle \cdot \ell=\mathrm{acl}_{1},\left\langle\mathrm{nd}_{j}, \mathrm{nd}_{k}\right\rangle} \exists \operatorname{linkR}\left(\mathrm{nd}_{j} \cdot \ell\right) \cdot C_{k} \sqcap \\
& \prod_{\left\langle\mathrm{nd}_{i}, \mathrm{nd}_{j}\right\rangle \cdot \ell \neq \mathrm{acl}} \exists \operatorname{mine}\left(\mathrm{nd}_{i} \cdot \ell, \mathrm{nd}_{j} \cdot \ell\right) \cdot C_{j}
\end{aligned}
$$

where $C_{k}$ and $C_{j}$ are the concepts corresponding to the sub-trees rooted at nodes $\mathrm{nd}_{k}$ and $\mathrm{nd}_{j}$, respectively.

Function mine is based on domain and range restrictions of properties. Consider for example nodes $\mathrm{nd}_{1}$ and $\mathrm{nd}_{2}$ in Fig. $1 \mathrm{~b}$ and assume that they have been 
linked to concepts Pain and Recent from SNOMED CT, respectively. Pain is a sub-concept of ClinicalFinding and Recent is a sub-concept of TemporalConcept. According to the SNOMED CT documentation, property temporalContext has these two concepts as a domain and range, respectively, so we can create concept Pain $\sqcap \exists$ temporalContext.Recent. If no property can be mined this way, mine returns assocWith. Modern KBs often come with such domain and range restrictions (e.g., DBpedia contains almost 2,000). If no such axioms exist then properties between concepts can be mined using, e.g., statistical approaches [19].

Our implementation of constructConcepts returns the concept corresponding to the root of the dependency tree and traverses it using a depth-first algorithm. Applied on the dependency tree of Fig. 1b, exploiting the domain and range restrictions in SNOMED CT and the existence of the verb in the tree, our method constructs concept Pain $\sqcap \exists$ temporalContext.Recent $\sqcap \exists$ causedBy.Injury.

If a dependency tree contains coordinating conjunctions, we split the dependency tree into multiple trees [31], construct a concept for each according to Definition 1 and connect them with conjunction to form an overall concept.

Even though the above method was initially developed as part of our hybrid reasoning framework (i.e. method constructConcepts in Algorithm 1), it can be used as a stand-alone approach for concept extraction from any phrase that follows a structure similar to concept labels. It can thus enable the usage of our hybrid reasoner with textual input queries to verify if these queries express the same information: first concepts are constructed from the queries using the standalone knowledge extraction component and subsequently the hybrid reasoner is applied as usual. This pipeline approach is further discussed in Sect.6. In the rest of this paper, we refer to our concept extraction method as concept builder.

\section{Practical Hybrid Reasoning over Large KBs}

Our hybrid reasoning approach presented in Sect. 3 uses logic-based reasoning to check subsumption between two given concepts (lines 1, 6, and 9 in Algorithm 1), which is implemented in SPARQL.

Unfortunately, the concepts we are dealing with may involve a number of conjuncts, existential quantifiers, or even be nested, while SPARQL cannot check subsumption recursively. To address these issues, we use a form of structural subsumption [3], which reduces subsumption checking to subsumption between the atomic elements of a (potentially complex) concept.

Definition 2. Let $C:=\prod_{i} A_{i} \sqcap \prod_{j=1}^{m} \exists R_{j} \cdot E_{j}$ and $D:=\prod_{k} B_{k} \sqcap \prod_{l=1}^{n} \exists S_{l} . F_{l}$ be two concepts and let $\mathcal{K}$ be a KB. We say that $C$ is structurally subsumed by $D$, denoted by $C \sqsubseteq_{s} D$, if and only if the following hold:

1. For every $B_{k}$ some $A_{i}$ exists such that $\mathcal{K} \models A_{i} \sqsubseteq B_{k}$.

2. for every $l \in[1, n]$ either of the following holds:

(a) there exists $j \in[1, m]$ s.t. $\mathcal{K} \models R_{j} \sqsubseteq S_{l}$ and $\mathcal{K} \models E_{j} \sqsubseteq s F_{l}$

(b) some $A_{i} \sqsubseteq \exists T . G \in \mathcal{K}$ exists s.t. $\mathcal{K} \models T \sqsubseteq S_{l}$ and $\mathcal{K} \models G \sqsubseteq s F_{l}$. 
If some conjunct $\exists S_{l} \cdot F_{l}$ of $D$ does not subsume any conjunct in $C$, condition $2 \mathrm{~b}$ performs an expansion on one of the main concepts $A_{i}$ of $C$, using the $\mathrm{KB}$ to check if $A_{i}$ is subsumed by some other concept that is subsumed by $\exists S_{l} \cdot F_{l}$. Definition 2 can be easily implemented as SPARQL queries over triple-stores.

Example 3. Consider a KB $\mathcal{K}$ containing the following axioms:

FootPain $\sqsubseteq \exists$ findingSite.Foot, FootPain $\sqsubseteq$ Pain, Foot $\sqsubseteq$ Limb

Let $C_{1}=$ FootPain and $C_{2}=$ Pain $\sqcap \exists$ findingSite.Limb and assume we want to check whether $\mathcal{K} \mid=C_{1} \sqsubseteq C_{2}$, which is indeed the case. According to Definition 2, $C_{1}$ is structurally subsumed by $C_{2}$ since $\mathcal{K} \models$ FootPain $\sqsubseteq$ Pain and FootPain $\sqsubseteq$ $\exists$ findingSite.Foot $\in \mathcal{K}$ such that $\mathcal{K} \models$ Foot $\sqsubseteq_{s} \operatorname{Limb}$ (condition $2 \mathrm{~b}$ of Definition 2).

As discussed in Sect.3, concept extraction and unfolding should only be applied in our hybrid reasoning framework if we have reason to believe that it will be helpful for proving a correct subsumption. Since our concept extraction method uses labels of concepts, we also base the instantiation of the applicability condition sim on the label similarity of the compared concepts $C$ and $D$.

Definition 3. Let concepts $C$ and $D$ of the same form as in Definition 2 be the input of Algorithm 1. Let str-sim be some string similarity algorithm and let $\oplus$ denote string concatenation. We instantiate $\operatorname{sim}(C, D)$ in line 2 of Algorithm 1 as $\operatorname{str}-\operatorname{sim}\left(\oplus_{i} \mathscr{L}\left(A_{i}\right) \oplus \oplus_{j=1}^{m} \mathscr{L}\left(E_{j}\right), \oplus_{k} \mathscr{L}\left(B_{k}\right) \oplus \oplus_{l=1}^{n} \mathscr{L}\left(F_{l}\right)\right)$.

For example, for concepts $C=$ FootPain and $D_{1}=$ Pain $\sqcap \exists$ findingSite.Head, the similarity score is expected to be lower compared to the one for $C$ and $D_{2}=$ Pain $\sqcap$ findingSite.Foot. Using an appropriate threshold $\sigma_{1}$, we can avoid applying concept builder on $C$ when we compare it to $D_{1}$ but do apply it when we compare it to $D_{2}$, so as to extract conjunct $\exists$ findingSite. Foot from label "Foot Pain" of $C$, which appears in $D_{2}$ and thus lets us prove subsumption. We apply Levenshtein distance for str-sim and find that setting $\sigma_{2}$ to half of the shorter string's length works well.

For the instantiation of the second applicability condition diff, we check whether the reason that subsumption failed was that some conjuncts in $D$ do not subsume any conjuncts in $C$.

Definition 4. Let $C, D$ be concepts of the same form as in Definition 2 and let $\mathcal{K}$ be a KB. Function $\operatorname{diff}(C, D)$ returns all $\exists S_{l} . F_{l}$ in $D$ such that both of the following hold:

1. $\forall j \in[1, m]$ either $\mathcal{K} \forall R_{j} \sqsubseteq S_{l}$ or $\mathcal{K} \forall \forall E_{j} \sqsubseteq s F_{l}$, and

2. no $A_{i} \sqsubseteq \exists T . G \in \mathcal{K}$ exists such that $\mathcal{K} \models T \sqsubseteq S_{l}$ and $\mathcal{K} \models G \sqsubseteq s F_{l}$

If the set returned has more than $\sigma_{2}$ elements (for our purpose $\sigma_{2}=0$ works well), then Algorithm 1 will try to extract conjuncts from $C$ that are subsumed by those in $D$ using concept builder in line 5 . An empty set expresses that the reason for non-subsumption of $C$ and $D$ is that $A$ is not subsumed by $B$, in which case $B$ rather than $A$ should be unfolded to be able to prove that $A$ is a more specific concept than $B$. 
Table 1. Examples of concepts constructed by concept builder for SNOMED labels

\begin{tabular}{l|l}
\hline Partially correct concept \\
\hline Blood in Urine & $\begin{array}{l}\text { Blood } \sqcap \exists \text { assocWith.Urine } \\
\text { Blood } \sqcap \exists \text { findingSite.Urine (doctor concept) }\end{array}$ \\
\hline Wrong concept & \\
\hline Prune belly syndrome & $\begin{array}{l}\text { Prune } \sqcap \text { } \text { assocWith.Syndrome } \sqcap \exists \text { findingSite.Belly } \\
\text { PruneBellySyndrome (doctor concept) }\end{array}$ \\
\hline
\end{tabular}

Example 4. Consider Example 1, where

$C_{1}:=$ RecentInjury $\sqcap \exists$ findingSite. Head

$C_{2}:=$ Injury $\sqcap \exists$ findingSite. Head $\sqcap \exists$ occurred.Recently

Then, $\operatorname{diff}_{\mathcal{K}}\left(C_{1}, C_{2}\right)=\{\exists$ occurred.Recently $\}$, which does not subsume any conjunct of similar form in $C_{1}$ and there is no such conjunct in the $\mathrm{KB}$ for RecentInjury either. Hence, Algorithm 1 will extract a concept from the label of $C_{1}$ as desired (if $\sigma_{2}=0$ ).

In contrast, diff $\mathcal{K}_{\mathcal{K}}\left(C_{2}, C_{1}\right)$ returns $\emptyset$ since the only conjunct of $C_{1}$ also exists in $C_{2}$ ( $\exists$ findingSite.Head). Thus, when calling isSubsumed ${ }_{\mathcal{K}}\left(C_{2}, C_{1}\right)$ the algorithm will skip the block in lines $4-7$ and will only apply concept builder on $C_{1}$.

\section{Evaluation}

Our work was motivated by the need to compare medical knowledge encoded in different services in Babylon Health, such as an AI-based symptom-checking chatbot, triaging, drug prescriptions, and telemedicine. Medical information in all services is encoded using (complex) concepts built from a large medical KB [4], curated from sources like SNOMED CT and NCI and stored in GraphDB [26].

Even though the same KB is used, different services (and even humans) may encode the same information in different ways. For example, concepts representing symptoms in the triaging system were manually created by doctors, encoding "swelling of ear" as an atomic KB concept SwollenEar. However, during a patient interaction with the chatbot the same phrase may be automatically encoded as Swelling $\sqcap \exists$ findingSite.Ear. To allow interoperability of services, a system able to identify these two concepts as being equivalent needs to be in place. We deploy our hybrid reasoner for this task.

In the following, we evaluate the performance of our hybrid reasoner on two different use cases that require subsumption reasoning. First, however, we evaluate the performance of concept builder in isolation, as it is an integral part of our hybrid reasoner and can be used as a stand-alone concept extraction tool.

\subsection{Performance of Concept Builder}

We randomly sampled 200 SNOMED CT concept labels containing at least two words and applied concept builder to extract (complex) concepts linked to 
Table 2. Number of equivalences and subsumptions proven by hybrid reasoner between complex/atomic doctors' concepts and supposedly equivalent concept builder concepts.

\begin{tabular}{l|l|l|l|l|l|r|r|r|l|r}
\hline \multirow{2}{*}{ Type of reasoning } & \multicolumn{9}{l|}{ Complex } & \multicolumn{1}{l|}{ Atomic } \\
\cline { 2 - 13 } & equiv & subs & none & error & timeout & equiv & subs & none & error & timeout \\
\hline Logic & 0 & 0 & 0 & 0 & 0 & 169 & 8 & 55 & 0 & 0 \\
+ expansion & 29 & 0 & 0 & 1 & 0 & 68 & 0 & 0 & 0 & 17 \\
+ builder & 36 & 4 & 0 & 0 & 0 & 411 & 68 & 67 & 2 & 2 \\
+ expansion/builder & 24 & 4 & 100 & 5 & 0 & 83 & 17 & 0 & 0 & 6 \\
\hline Total & 89 & 8 & 100 & 6 & 0 & 731 & 93 & 122 & 2 & 25 \\
\hline
\end{tabular}

the KB. Three doctors then evaluated the quality of concepts constructed by concept builder by classifying each as correct, partially correct, or wrong. Rare disagreements between doctors' judgements were resolved by discussions.

For 19 labels, concept builder failed to extract a concept from the SNOMED label as it was unable to link some of the words in the label to KB concepts. For the remaining 181 SNOMED labels concept builder exhibits good performance, with $60 \%$ of extracted concepts evaluated as correct by doctors, $29 \%$ as partially correct, and only $11 \%$ as wrong. As illustrated in Table 1, the most common reasons for concepts being wrong or only partially correct are an incorrect choice of property (first example in the table) and insufficient tree pruning, resulting in too many atomic concepts in the constructed concept (second example in the table). Overall, we consider the performance of our concept builder sufficiently good to be applied as the knowledge extraction component in our hybrid reasoner and as a stand-alone tool for constructing concepts from short phrases.

\subsection{Use Case 1: Constructing the Symptom-Checking Network}

The first dataset to evaluate our hybrid reasoner is based on a symptom-checking engine, consisting of a network of 1176 nodes representing symptoms, diseases, and risk factors interconnected with probabilities. A group of doctors created this network manually by associating each medical entity node they wanted the network to contain with an atomic or complex concept based on the KB. For example, the doctors created a node for "Aching Epigastric Pain" and associated it with the concept EpigastricPain $\sqcap$ GhasSensation.AchingSensation. Besides the concept, the group of doctors also stored the name of the medical entity they represented.

To test how well our hybrid reasoner can identify equivalent concepts, we apply our concept builder on the name associated with each node to automatically obtain a second concept representation $D$ using the KB. Since $D$ is based on the same entity name as concept $C$ constructed by doctors, $C$ and $D$ should be equivalent. We use the hybrid reasoner to check this, i.e. if $C \sqsubseteq D$ and $D \sqsubseteq C$, giving an insight into how well the hybrid reasoner can identify equivalences. 
Table 3. Examples and reasons why our hybrid reasoner could not prove equivalence between two concepts (top concept - concept builder; bottom concept - doctor).

\begin{tabular}{|c|c|}
\hline \multicolumn{2}{|c|}{ Semantic equivalence } \\
\hline Poor hygiene & $\begin{array}{l}\text { Hygiene } \sqcap \text { ヨassociatedWith.Poor } \\
\text { NeglectOfPersonalHygiene }\end{array}$ \\
\hline \multicolumn{2}{|c|}{ Highly complex concept } \\
\hline $\begin{array}{l}\text { Analgesia overuse } \\
\text { headache }\end{array}$ & $\begin{array}{l}\text { Headache } \Pi \exists \text { hasDef.Analgesia } \Pi \\
\quad \exists \text { assocWith.RepetitiveStrainInjury } \\
\text { AnalgesicOveruseHeadache }\end{array}$ \\
\hline
\end{tabular}

Our results are summarised in Table 2, distinguishing between atomic and complex concepts created by doctors. The columns "equiv" and "subs" denote cases where both or only one of the above subsumption calls to the reasoner succeeded. Column "none" counts concepts for which our hybrid reasoner could not prove subsumption, "error" denotes cases in which concept builder failed to construct a concept for the given phrase, and "timeout" cases in which the reasoner exceeded the time-out limit of five seconds. Rows distinguish the type of reasoning performed by the reasoner while trying to prove the two subsumption directions, in particular, simple logic-based reasoning, i.e. no expansion and no concept extraction (line 1 in Algorithm 1 without using $2 \mathrm{~b}$ in Definition 2), logic-based reasoning with concept expansion (line 1 in Algorithm 1 using 2b in Definition 2), and logic-based reasoning (and concept expansion) with the application of concept builder on KB labels (Algorithm 1 past line 1). The last row, expansion/builder, denotes cases where one subsumption direction required expansion (without concept builder) and the other direction also required the usage of concept builder.

Table 2 shows that our hybrid reasoner exhibits good performance: in $70 \%$ of the cases it can prove the equivalence of concepts and in another $9 \%$ it can prove that one is subsumed by the other. Concept expansion has a significant impact in increasing the inference power of determining subsumption. Furthermore, applying concept builder for on-the-fly knowledge extraction from concept labels leads to another huge improvement. Note that for complex concepts, pure logic-based reasoning is unable to prove any equivalences, illustrating the usefulness of our hybrid reasoner.

Further analysis revealed that logic-based reasoning is only able to prove equivalence if the two given concepts $C$ and $D$ are in fact the same atomic concept $A$, i.e. $C=A$ and $D=A$. In contrast, in all cases in which our hybrid reasoner applied knowledge extraction and expansion to prove equivalence, at least one of the given concepts was a complex concept, making the reasoning much more challenging. Note that for any complex concept by a doctor, concept builder constructed a different complex concept, making subsumption checking extremely difficult. Despite this complexity, our hybrid reasoner is able to prove equivalence in half of these highly challenging cases. 
The main reasons why our reasoner failed to prove subsumption between concepts was that the two concepts were semantically equivalent but expressed using completely different concepts and that one concept was way more complex than the other, as illustrated in Table 3. Overall, this use case shows the advantage of our hybrid subsumption reasoning method, which applies knowledge extraction to tackle the inherent incompleteness in $\mathrm{KBs}$, as compared to pure logic-based reasoning.

\subsection{Use Case 2: Understanding User Queries for Symptom-Checking}

The symptom-checking engine from the first use case is accessed by end-users through a chatbot. Given a user query such as "My stomach hurts", the correct node from the network needs to be activated to initiate symptom-checking.

Before the development of our hybrid reasoner, two different methods were tested to map user queries to nodes in the network. First, the GATE text annotation system [7], which applies string matching and hand-crafted rules, was used to identify names of symptom nodes in a query. Second, the Google universal sentence embedder [5] (based on Transformer) was used to embed both the user query and all symptom nodes in the network and then the node with the closest vector compared to the query vector (using cosine similarity) was chosen.

Our hybrid reasoner can be used to map queries to nodes as follows: first, concept builder extracts a concept $C$ from user text, then the hybrid reasoner finds a "closest" matching symptom by checking which node in the network is associated with a concept $D$ that subsumes $C$, i.e. for which $D$ it holds that $C \sqsubseteq D$. We refer to this pipeline as ConBReas(Concept Builder-Reasoner).

To systematically compare the performance of the three methods, doctors created a dataset of 1878 mock user queries ${ }^{2}$ and matched each with a node from the network. For example, doctors matched the query "I can't focus" to the node associated with concept PoorConcentration. In addition to comparing the performance of GATE and the embedder to ConBReas, we also experiment with using the embedder as a fallback in GATE and ConBReas, that is, if either of these approaches fails to return a node from the network then the embedder is used; we call these settings GATE $_{\mathrm{emb}}$ and ConBReasemb, respectively.

Table 4. Performance of different methods on our query-symptom dataset.

\begin{tabular}{l|l|l|l|l|l}
\hline & GATE & GATE $_{\text {emb }}$ & emb & ConBReas & ConBReas \\
\hline Precision & 1.00 & 0.86 & 0.72 & 0.96 & 0.84 \\
Recall & 0.53 & 0.74 & 0.72 & 0.79 & 0.88 \\
\hline
\end{tabular}

$\overline{{ }^{2} \text { For privacy }}$ reasons we do not use real user queries. 
Table 5. Examples of false positives (FP) and false negatives (FN) of ConBReas.

\begin{tabular}{l|l|l|l}
\hline User query & ConBReas & Correct symptom & Type \\
\hline I don’t sleep well & SleepingWell & RestlessSleep & FP \\
I'm hungry all the time & Always $\sqcap \exists$ assocWith.Hungry & IncreasedAppetite & FP \\
I can’t speak properly & - & DifficultySpeaking & FN \\
I'm getting skinnier & - & WeightLoss & FN \\
\hline
\end{tabular}

Performance results of the different methods are given in Table 4. As expected, GATE offers the lowest recall but highest precision, since it performs an almost exact lexical match between the words in a query and the possible symptom labels. The combination of GATE with an embedder increases its recall, but decreases precision. The embedder provides better recall but the precision is too low for a medical application. ConBReas outperforms the embedder in terms of both precision and recall. Compared to GATE, ConBReas' precision

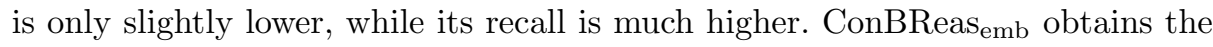
best trade-off between precision and recall among all methods, and is thus used in production to link user queries to the symptom checking engine.

For future improvement, we performed an error analysis and found that most of ConBReas' mistakes are due to concept construction from the user query, and in particular the entity linking step if sophisticated common-sense reasoning is needed to link the correct concept. Table 5 gives some examples of false positives (wrong concept constructed) and false negatives (no concept constructed).

\section{Related Work and Conclusions}

To the best of our knowledge, our hybrid reasoning framework is the first approach applying statistical methods as part of logic-based subsumption checking. Conversely, Movshovitz-Attias et al. [18] train a subsumption classifier and include logic-based features using a KB, in particular the overlap of concepts' properties in Biperpedia. Like us they exploit dependency trees, used in terms of a feature expressing whether any paths in the dependency trees of the two concepts match. In contrast to our hybrid reasoner, the results of their classifier are not easily explainable and the classifier needs sufficient training data, whereas our reasoner can be applied without training and can be easily interpreted.

The problem of learning DL axioms from unstructured text has received considerable attention $[8,11,22,28]$. The structure of text targeted by these works is substantially different to the one usually found in concept labels. These works deal with verb phrases which often have some definitory character, e.g. from text "Enzymes are proteins that catalyse chemical reactions" an axiom of the form Enzyme $\sqsubseteq$ Protein $\sqcap \exists$ catalyse.ChemicalReaction can be extracted. In contrast, concept labels - as used by us - are usually short phrases without verbs, so properties between entities need to be inferred as they cannot be extracted from the text. The work by Romacker [25] is close to ours as they also use 
dependency parsing and then map the dependency tree to DL concepts. However, again full sentences are targeted using a form of reification. For our running example (Fig. 1a) their approach would create Provoking $\sqcap$ provokingAgent.Injury $\square$ $\exists$ provokingPatient.Pain, whereas ours produces simpler and more concise concepts enabling easier subsumption checking.

Concept labels have been previously proven useful for extracting additional information from KBs [9,21]. Fernandez-Breis et al. [9] use manually created patterns to "decompose" concept labels, whereas Pacheco et al. [21] use all subwords in a concept label and do not consider any dependency relations between them. Our approach is less rigid as it construct concepts dynamically based on the structure of the dependency tree.

The structure of concept labels found in KBs resembles keyword-based and entity-oriented natural language queries $[13,17,23]$. Compared to Pound et al. [23] we can extract arbitrary (even nested) existentially quantified concepts and do not require training of application-specific template classifiers. Furthermore, we do not require that relations between concepts are expressed by words in the query [13]. Similar to us, Lei et al. [17] derive KB properties to connect concepts from words in the query or, if not present, from the KB structure. However, they construct a tree from the query which needs to be a sub-tree of the $\mathrm{KB}$, whereas our complex concepts are not tied to the KB structure in this way. $\mathrm{Xu}$ et al. [30] use dependency trees for relation extraction, however they require task-specific training data, whereas we apply pre-trained dependency parsers.

The comparison with related work highlights the novelty of our hybrid reasoning framework in integrating knowledge extraction from concept labels using NLP methods into logic-based subsumption checking. We have provided an instantiation of our hybrid reasoning framework paying extra care on design choices in order to provide a highly efficient system that can operate in a production environment. Our evaluation on two industrial use cases showed that our approach provides the best combination of precision and recall compared to purely statistical or purely rule-based approaches and our reasoner is thus currently employed in an industrial production environment.

Although our use case and implementation regards the medical domain, all methods are domain independent and can be adapted easily to new domains. For example, concept builder can be used on phrases like "Italian Painters" over DBpedia to construct concept Painter $\sqcap \exists$ birthPlace.Italy or on "Thriller Movies" over the Movie $\mathrm{KB}^{3}$ to construct concept Movie $\square$ ヨbelongsToGenre.Thriller. Future work will target better compounding methods in concept builder to avoid the construction of over-complicated concepts as well as the integration of common-sense reasoning methods to infer equivalence in difficult cases.

\footnotetext{
${ }^{3}$ http://www.movieontology.org/.
} 


\section{References}

1. Auer, S., Bizer, C., Kobilarov, G., Lehmann, J., Cyganiak, R., Ives, Z.: DBpedia: a nucleus for a web of open data. In: Aberer, K., et al. (eds.) ASWC/ISWC 2007. LNCS, vol. 4825, pp. 722-735. Springer, Heidelberg (2007). https://doi.org/10. 1007/978-3-540-76298-0_52

2. Baader, F., Brandt, S., Lutz, C.: Pushing the EL envelope. In: IJCAI, pp. 364-369 (2005)

3. Baader, F., Calvanese, D., McGuinness, D.L., Nardi, D., Patel-Schneider, P.F. (eds.): The Description Logic Handbook: Theory, Implementation, and Applications. Cambridge University Press, Cambridge (2003)

4. Barisevičius, G., et al.: Supporting digital healthcare services using semantic web technologies. In: Vrandečić, D., et al. (eds.) ISWC 2018. LNCS, vol. 11137, pp. 291-306. Springer, Cham (2018). https://doi.org/10.1007/978-3-030-00668-6_18

5. Cer, D., et al.: Universal sentence encoder. CoRR abs/1803.11175 (2018)

6. Choi, J.D., McCallum, A.: Transition-based dependency parsing with selectional branching. In: ACL, pp. 1052-1062 (2013)

7. Cunningham, H., Tablan, V., Roberts, A., Bontcheva, K.: Getting more out of biomedical documents with gate's full lifecycle open source text analytics. PLoS Comput. Biol. 9(2), e1002854 (2013)

8. Distel, F., Ma, Y.: A hybrid approach for learning SNOMED CT definitions from text. In: DL, pp. 156-167 (2013)

9. Fernandez-Breis, J.T., Iannone, L., Palmisano, I., Rector, A.L., Stevens, R.: Enriching the gene ontology via the dissection of labels using the ontology pre-processor language. In: Cimiano, P., Pinto, H.S. (eds.) EKAW 2010. LNCS (LNAI), vol. 6317, pp. 59-73. Springer, Heidelberg (2010). https://doi.org/10.1007/978-3-642-16438$5 \_5$

10. Galárraga, L., Razniewski, S., Amarilli, A., Suchanek, F.M.: Predicting completeness in knowledge bases. In: WSDM, pp. 375-383 (2017)

11. Gyawali, B., Shimorina, A., Gardent, C., Cruz-Lara, S., Mahfoudh, M.: Mapping natural language to description logic. In: Blomqvist, E., Maynard, D., Gangemi, A., Hoekstra, R., Hitzler, P., Hartig, O. (eds.) ESWC 2017. LNCS, vol. 10249, pp. 273-288. Springer, Cham (2017). https://doi.org/10.1007/978-3-319-58068-5_17

12. Hachey, B., Radford, W., Nothman, J., Honnibal, M., Curran, J.R.: Evaluating entity linking with wikipedia. Artif. Intell. 194, 130-150 (2013)

13. Han, S., Zou, L., Yu, J.X., Zhao, D.: Keyword search on RDF graphs - a query graph assembly approach. In: CIKM, pp. 227-236 (2017)

14. Hou, J., Nayak, R.: A concept-based retrieval method for entity-oriented search. In: AusDM, pp. 99-105 (2013)

15. Kazakov, Y., Krötzsch, M., Simancik, F.: The incredible ELK - from polynomial procedures to efficient reasoning with $\mathcal{E} \mathcal{L}$ ontologies. J. Autom. Reason. 53(1), 1-61 (2014)

16. Kübler, S., McDonald, R.T., Nivre, J.: Dependency Parsing. Synthesis Lectures on Human Language Technologies. Morgan \& Claypool Publishers, San Francisco (2009)

17. Lei, C., et al.: Ontology-based natural language query interfaces for data exploration. IEEE Data Eng. Bull. 41(3), 52-63 (2018)

18. Movshovitz-Attias, D., Whang, S.E., Noy, N.F., Halevy, A.Y.: Discovering subsumption relationships for web-based ontologies. In: WebDB, pp. 62-69 (2015) 
19. Nickel, M., Murphy, K., Tresp, V., Gabrilovich, E.: A review of relational machine learning for knowledge graphs. Proc. IEEE 104(1), 11-33 (2016)

20. Oramas, S., Ostuni, V.C., Noia, T.D., Serra, X., Sciascio, E.D.: Sound and music recommendation with knowledge graphs. ACM TIST 8(2), 21:1-21:21 (2016)

21. Pacheco, E.J., Stenzhorn, H., Nohama, P., Paetzold, J., Schulz, S.: Detecting under specification in SNOMED CT concept definitions through natural language processing. In: AMIA (2009)

22. Petrova, A., et al.: Formalizing biomedical concepts from textual definitions. J. Biomed. Semant. 6(1), 22 (2015). https://doi.org/10.1186/s13326-015-0015-3

23. Pound, J., Hudek, A.K., Ilyas, I.F., Weddell, G.: Interpreting keyword queries over web knowledge bases. In: CIKM, pp. 305-314 (2012)

24. Raiman, J., Raiman, O.: DeepType: multilingual entity linking by neural type system evolution. In: AAAI, pp. 5406-5413 (2018)

25. Romacker, M., Markert, K., Hahn, U.: Lean semantic interpretation. In: IJCAI, pp. 868-875 (1999)

26. Stoilos, G., Geleta, D., Shamdasani, J., Khodadadi, M.: A novel approach and practical algorithms for ontology integration. In: ISWC (2018)

27. Stuckenschmidt, H., Ponzetto, S.P., Meilicke, C.: Detecting meaningful compounds in complex class labels. In: Blomqvist, E., Ciancarini, P., Poggi, F., Vitali, F. (eds.) EKAW 2016. LNCS (LNAI), vol. 10024, pp. 621-635. Springer, Cham (2016). https://doi.org/10.1007/978-3-319-49004-5_40

28. Völker, J., Hitzler, P., Cimiano, P.: Acquisition of OWL DL axioms from lexical resources. In: Franconi, E., Kifer, M., May, W. (eds.) ESWC 2007. LNCS, vol. 4519, pp. 670-685. Springer, Heidelberg (2007). https://doi.org/10.1007/978-3540-72667-8_47

29. Wessel, M., Acharya, G., Carpenter, J., Yin, M.: OntoVPA — an ontology-based dialogue management system for virtual personal assistants. In: Eskenazi, M., Devillers, L., Mariani, J. (eds.) Advanced Social Interaction with Agents. LNEE, vol. 510, pp. 219-233. Springer, Cham (2019). https://doi.org/10.1007/978-3-31992108-2_23

30. Xu, H., Hu, C., Shen, G.: Discovery of dependency tree patterns for relation extraction. In: PACLIC, pp. 851-858 (2009)

31. Zhang, W., Liu, S., Yu, C., Sun, C., Liu, F., Meng, W.: Recognition and classification of noun phrases in queries for effective retrieval. In: CIKM, pp. 711-720 (2007) 